International Journal of Engineering \& Technology, $7(4.5)(2018) 410-415$
International Journal of Engineering \& Technology
SPC
Website: www.sciencepubco.com/index.php/IJET
Research paper

\title{
Viscous Dissipation and Dufour Effects on MHD Free Convection Flow Through an Oscillatory Inclined Porous Plate with Hall and Ion-Slip Current
}

\author{
K.V. B. Raja kumar ${ }^{1 *}$ K.S. Balamurugan ${ }^{2}$ Ch. V. Ramana Murthy ${ }^{3} \quad$ N.Ranganath $^{4}$ \\ ${ }^{1}$ Department of Mathematics, Kallam Haranadhareddy Institute of technology Andhra Pradesh, India \\ ${ }^{2}$ Department of Mathematics, RVR\&JC College of Engineering, Guntur, Andhra Pradesh, India \\ ${ }^{3}$ Department of Mathematics, Sri Vasavi Institute of Engineering \& Technology, Nanadamuru (AP), India \\ ${ }^{4}$ Department of Mathematics, Shinas college of Technology, Muscat Oman. \\ *Corresponding AuthorEmail:kvbrajakumar@gmail.com
}

\begin{abstract}
In this paper the viscous dissipation and Dufour effects on Unsteady MHD free convective flow through a semi-infinite Oscillatory porous inclined plate of time dependent permeability with Chemical reaction and Hall and Ion-Slip Current in a Rotating System was investigated. The dimensionless governing equations for this investigation are solved analytically by using multiple regular perturbation law. The effects of different parameters on velocity, temperature and concentration fields are shown graphically.
\end{abstract}

Keywords: chemical reaction; Dufour; Hall Effect; Ion-Slip effects; Radiation absorption; viscous dissipation

\section{Introduction}

Magneto hydrodynamic flow of heat and mass transfer processes occur in many of the industrial applications: such as cooling of geothermal systems, aerodynamic processes, chemical catalytic reactors and processes, electromagnetic pumps, and Magneto Hydrodynamic power generators etc Viscous dissipation expects a basic part in changing the temperature movement, much the same as an imperativeness source, which impacts the heat transfer rates fundamentally. Shankar Goud et al. [1] analyzed of viscous dissipation and diffusion thermo on effects on unsteady Magneto hydrodynamic flow past an impulsively started inclined oscillating plate with mass diffusion and variable temperature. Alivene et al. [2] examined the viscous dissipation, radiation and Hall effects on convective heat and mass transfer flow of a fluid past a stretching sheet. Subhakanthi et al. [3] Analyzed the viscous dissipation and chemical reaction effects on heat and mass transfer in Magneto Hydrodynamic flow past an inclined porous plate. G.V. Reddy et al. [4] reported viscous dissipation and radiation effects on unsteady Magneto hydrodynamic free convection heat and mass transfer flow past a semi-infinite inclined moving plate. Rajput $e t$ al [5] considered effects of chemical reaction and Hall current on unsteady Magneto Hydrodynamic flow past through an oscillating inclined porous plate with mass diffusion and variable temperature. Rajput et al [6] examined the effects of Hall current and chemical reaction on unsteady Magneto Hydrodynamic flow past an impulsively started inclined plate with mass diffusion and variable wall temperature. The energy flux caused due to composition gradient is known as Dufour effect. Raj put et al [7] Hall effects on unsteady Magneto Hydrodynamic flow past over exponentially accelerated inclined porous plate with mass transfer and variable wall temperature was analyzed. In the investigation Dufour effect was not consider. Jithender Reddy et al [8] considered Dufour, thermal radiation and hall current on unsteady magneto hydrodynamic natural convective heat and mass transfer of a fluid flow past an impulsively moving vertical porous plate in the presence of ramped temperature. Ion-slip and Hall current are probably going to be fundamental in flows of lab plasma when a solid magnetic field of a uniform quality is connected and drawn the consideration of the analysts MD. Shah Alam et al [9]Magneto Hydrodynamic free convection heat and mass transfer of fresh as well as salt water flow on an infinite inclined plate with Hall current and constant heat flux. Sivaiah et al [10] studied hall current and radiation effects on Magneto Hydrodynamic free convective flow past an inclined porous plate with thermal diffusion and heat source. Remembering every one of these realities in this work the effect the Dufour, Radiation absorption, Chemical reaction, and viscous dissipation on Unsteady MHD free convective flow through a semi-infinite vertical Oscillatory inclined porous plate of time dependent permeability with Hall and Ion-Slip Current in a Rotating System was investigated. The perturbation technique is employed to solve governing coupled non-liner partial differential equations.

\section{Mathematical Formulation}

The unsteady flow of a electrically conducting incompressible viscous fluid past semi-infinite inclined porous plate $\mathrm{y}=0$ has been considered, with the $\mathrm{x}$-axis chosen along the plate, when the plate velocity $U(t)$ oscillates in $t$ with a frequency $n$ and is given as $\mathrm{U}(\mathrm{t})=\mathrm{U}_{0}(1+\cos n t)$.Let the $\mathrm{x}^{*}$ and $\mathrm{y}^{*}$ are the dimensional distance along the perpendicular to the plate and $t^{*}$ is the time. The physical model of the flow problem is shown in figure A. $\mathrm{u}^{*}$ is the component of dimensional velocities along $\mathrm{x}^{*}$ and $\mathrm{y}^{*}$ directions. The flow is assumed to be in $\mathrm{x}$-direction and which is taken along 
the plate in upward direction and y-axis is normal to it. Initially the fluids as well as the plate are at rest but for time $t>0$ the whole system is allowed to rotate with a constant angular velocity $\Omega$ about the y-axis. Assumed transverse magnetic field of the uniform strength $\mathrm{B}_{0}$ is to be utilizable normal to the plate. Viscous dissipation, Radiation absorption, the heat source, and Dufour effects are considered. The physical configuration of the problem is shown in the Figure A.

Hence dimensional governing equations are;

Equation of Momentum:

$\left[\frac{\partial u^{*}}{\partial \tau^{*}}\right]=\vartheta\left[\frac{\partial^{2} u^{*}}{\partial y^{*^{2}}}\right]+g \beta\left[\left(T^{*}-T_{\infty}^{*}\right) \cos \psi+\frac{\beta^{*}}{\beta}\left(C^{*}-C_{\infty}^{*}\right) \cos \psi\right]$

$+2 \Omega w^{*}-\frac{v}{k^{*}}\left[u^{*}\right]-\frac{B_{0}^{2} \sigma_{e}\left[\alpha_{e} u^{*}+\beta_{e} w^{*} \cos \psi\right] \cos ^{2} \psi}{\rho\left[\alpha_{e}^{2}+\beta_{e}^{2} \cos \psi\right]}$

$\left[\frac{\partial w^{*}}{\partial \tau^{*}}\right]=\vartheta\left[\frac{\partial^{2} w^{*}}{\partial y^{*}}\right]-2 \Omega\left[u^{*}\right]-\frac{\vartheta}{k^{*}}\left[w^{*}\right]$

$$
+\frac{B_{0}^{2} \sigma_{e}\left[\beta_{e} u^{*} \cos \psi-\alpha_{e} w^{*}\right] \cos ^{2} \psi}{\rho\left[\alpha_{e}^{2}+\beta_{e}^{2} \cos \psi\right]}
$$

Equation of Energy:

$\left[\frac{\partial T^{*}}{\partial \tau^{*}}\right]=\frac{K}{\rho C_{p}}\left[\frac{\partial^{2} T^{*}}{\partial y^{*}}\right]+\frac{D_{m} K_{T}}{C_{S} C_{P}}\left[\frac{\partial^{2} C^{*}}{\partial y^{*}}\right]+\frac{Q_{0}}{\rho C_{p}}\left[T^{*}-T_{\infty}^{*}\right]$
$-\frac{1}{k \rho C_{p}}\left[\frac{\partial q_{r}^{*}}{\partial y^{*}}\right]+\frac{\vartheta}{C}\left[\left(\frac{\partial u^{*}}{\partial y^{*}}\right)^{2}\left(\frac{\partial w^{*}}{\partial y^{*}}\right)^{2}\right]+R^{*}\left[C^{*}-C_{\infty}^{*}\right]$

Concentration species diffusion equation:

$\left[\frac{\partial C^{*}}{\partial \tau^{*}}\right]=D_{m}\left[\frac{\partial^{2} C^{*}}{\partial y^{*^{2}}}\right]-K_{r}\left[C^{*}-C_{\infty}^{*}\right]$

The initial and boundary conditions are as

$\left.\begin{array}{l}\text { at } y^{*}=0 \Rightarrow\left\{\begin{array}{l}u^{*}=U_{0}\left[1+\frac{\varepsilon}{2}\left(e^{i \mathrm{n}^{*}}+e^{-i \mathrm{n}^{*} \mathrm{t}}\right)\right], w^{*}=0, \\ T^{*}=T_{w}^{*}+\varepsilon\left(T_{w}^{*}-T_{\infty}^{*}\right) e^{i t^{*}}, \\ C^{*}=C_{w}^{*}+\varepsilon\left(C_{w}^{*}-C_{\infty}^{*}\right) e^{i t^{*}}\end{array}\right. \\ \text { as } y^{*} \rightarrow \infty u^{*}=0, w^{*}=0, T^{*}=T_{\infty}^{*}, C^{*}=C_{\infty}^{*}\end{array}\right\}$

Using the relation in the radiative heat flux $\left(q_{r}\right)$ for the optically thin non gray gas near equilibrium is given by

$\frac{\partial q_{r}}{\partial y}=4 I^{1} T\left[T-T_{1}\right], \quad I^{1}=\int_{0}^{\infty} K_{\lambda_{1} w} \frac{\partial e_{b \lambda_{1}}}{\partial T} d \lambda_{1}$ Where $q_{r}$ is the

radiative heat flux, $K_{\lambda_{1} w}$ is the radiation absorption coefficient at the wall and $e_{b \lambda_{1}}$ is Plank's constant. The permeability of the porous medium is assumed to be $k^{*}=k_{0}\left[1+\varepsilon e^{-n t}\right]$, here $k_{0}$ is the constant permeability of the medium. Introducing the following non-dimensional quantities in the (1)-(4)

$$
\left.\begin{array}{l}
U_{0} u=u^{*}, U_{0} w=w^{*}, \vartheta y=y^{*} U_{0}, t \vartheta=U_{0}^{2} \tau^{*}, \\
n U_{0}^{2}=\vartheta n^{*}, T^{*}-T_{\infty}^{*}=\left(T_{w}^{*}-T_{\infty}^{*}\right) \theta, \\
C^{*}-C_{\infty}^{*}=\left(C_{w}^{*}-C_{\infty}^{*}\right) \phi,
\end{array}\right\}
$$

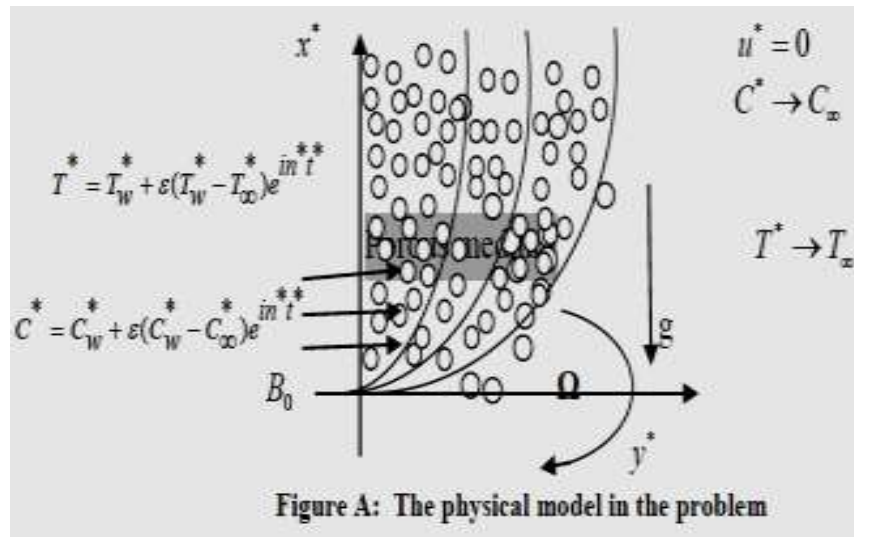

Then the equation (1), (2), (3) and (4) reduce to the following nondimensional form of equations

$$
\begin{aligned}
& {\left[\frac{\partial u}{\partial t}\right]=\left[\frac{\partial^{2} u}{\partial y^{2}}\right]+G_{r}[\theta] \cos \psi+G_{m}[\phi] \cos \psi+2 R[w]} \\
& -\gamma[u]-\frac{M\left[\alpha_{e} u+\beta_{e} w \cos \psi\right] \cos ^{2} \psi}{\left[\alpha_{e}^{2}+\beta_{e}^{2} \cos \psi\right]}
\end{aligned}
$$

$$
\begin{aligned}
& {\left[\frac{\partial u}{\partial t}\right]=\left[\frac{\partial^{2} u}{\partial y^{2}}\right]+G_{r}[\theta] \cos \psi+G_{m}[\phi] \cos \psi+2 R[w]} \\
& -\gamma[u]-\frac{M\left[\alpha_{e} u+\beta_{e} w \cos \psi\right] \operatorname{Cos}^{2} \psi}{\left[\alpha_{e}^{2}+\beta_{e}^{2} \cos \psi\right]}
\end{aligned}
$$

$$
\begin{array}{r}
{\left[\frac{\partial \theta}{\partial t}\right]=\frac{1}{\operatorname{Pr}}\left[\frac{\partial^{2} \theta}{\partial y^{2}}\right]-N[\theta]+D r\left[\frac{\partial^{2} \phi}{\partial y^{2}}\right]+R_{a}[\phi]} \\
+E c\left(\left(\frac{\partial u^{*}}{\partial y^{*}}\right)^{2}+\left(\frac{\partial w^{*}}{\partial y^{*}}\right)^{2}\right)
\end{array}
$$

$$
\left[\frac{\partial \phi}{\partial t}\right]=(S c)^{-1}\left[\frac{\partial^{2} \phi}{\partial y^{2}}\right]-K_{r}[\phi]
$$


$\xi=\frac{\vartheta Q_{0}}{\rho U_{0}^{2} C_{P}}, M=\frac{\sigma_{e} B_{0}^{2} \vartheta}{\rho U_{0}^{2}}, G_{m}=\frac{\vartheta \beta^{*} g\left[C_{w}^{*}-C_{\infty}^{*}\right]}{U_{0}^{2}}$

$, G_{r}=\frac{\vartheta \beta g\left[T_{w}^{*}-T_{\infty}^{*}\right]}{U_{0}^{2}}, \eta=\frac{4 \vartheta I^{\prime}}{K_{p} C_{p} U_{0}^{2}}, R=\frac{\Omega \vartheta}{U_{0}^{2}}$

$S c=\frac{\vartheta}{D_{m}}, R_{a}=\frac{R^{*} \vartheta\left[C_{w}^{*}-C_{\infty}^{*}\right]}{U_{0}^{2}\left[T_{w}^{*}-T_{\infty}^{*}\right]}, \operatorname{Pr}=\frac{\rho \vartheta C_{p}}{\sigma}$,

$D r=\frac{D_{m} K_{T}\left[C_{w}^{*}-C_{\infty}^{*}\right]}{\vartheta C_{S} C_{P}\left[T_{w}^{*}-T_{\infty}^{*}\right]}, \alpha_{e}=1+\beta_{e} \beta_{i}, \gamma=\frac{\vartheta^{2}}{k^{*} U_{0}^{2}}$

$E c=\frac{U_{0}^{2}}{C_{p}\left[T_{w}^{*}-T_{\infty}^{*}\right]}, K_{r}=\frac{k_{1} \vartheta}{V_{0}^{2}}, N=[\xi+\eta]$,

$\lambda=\left[[2 R i+\gamma]+\frac{M\left[-\alpha_{e}+i \beta_{e} \cos \psi\right] \cos ^{2} \psi}{\left[\alpha_{e}^{2}+\beta_{e}^{2} \cos \psi\right]}\right]$,

Equations (9) - (10) are displayed, in a reduced form, as

$\frac{\partial U}{\partial t}=\frac{\partial^{2} U}{\partial y^{2}}+G_{r} \theta \cos \psi+G_{m} \phi \cos \psi-\lambda U$

Where $U=u+i w$

$\left.\begin{array}{l}\text { At } y=0 \quad U=\left[1+\frac{\varepsilon}{2}\left(e^{i \mathrm{nt}}+e^{-i \mathrm{nt}}\right)\right]=1, \theta=1+\varepsilon e^{i n t}, \phi=1+\varepsilon e^{i n t} \\ \text { As } y \rightarrow \infty \quad F \rightarrow 0, \theta \rightarrow 0, \phi \rightarrow 0\end{array}\right\}$

\section{Method of Solution:}

The resulting system of nonlinear ODEs Esq. (11), (12) and (14) subject to the boundary conditions presented in Esq. (12) has been explored numerically through Multiple Regular Perturbation law.

$$
\left.\begin{array}{l}
F=F_{0}(y)+\varepsilon e^{i n t} F_{1}(y)+o\left(\varepsilon^{2}\right) \ldots \ldots \\
\theta=\theta_{0}(y)+\varepsilon e^{i n t} \theta_{1}(y)+o\left(\varepsilon^{2}\right) \ldots \ldots \\
\phi=\phi_{0}(y)+\varepsilon e^{i n t} \phi_{1}(y)+o\left(\varepsilon^{2}\right) \ldots \ldots
\end{array}\right\}
$$

After Substitute (14) in the equations (9), (10) and (12) then we get

$$
\begin{aligned}
& U_{0}{ }^{\prime \prime}-\lambda U_{0}=-G_{r} \cos \psi \theta_{0}-G_{m} \cos \psi \phi_{0} \\
& U_{1}^{\prime \prime}-[\lambda+n i] U_{1}=-G_{r} \cos \psi \theta_{1}-G_{m} \cos \psi \phi_{1} \\
& \theta_{1}^{\prime \prime}-\operatorname{Pr}[N+i n] \theta_{1}=-\operatorname{Pr} \operatorname{Dr} \phi_{1}^{\prime \prime}-2 \operatorname{Pr} E c F_{0}^{\prime} F_{1}^{\prime}-\operatorname{Pr} R_{a} \phi_{1} \\
& \theta_{0}^{\prime \prime}-\operatorname{Pr} N \theta_{0}=-\operatorname{Pr} \operatorname{Dr}_{0}-\operatorname{Pr} E c\left[U_{0}^{\prime}\right]\left[U_{0}^{\prime}\right]-\operatorname{Pr} \operatorname{Ra} \phi_{0} \\
& \phi_{0}^{\prime \prime}-S_{c} K_{r} \phi_{0}=0 \\
& \phi_{1}^{\prime \prime}-S_{c}\left[K_{r}+n\right] \phi_{1}=0
\end{aligned}
$$

The corresponding boundary conditions can be written as

$$
\left.\begin{array}{r}
U_{0}=1, U_{1}=0, \theta_{0}=1 \theta_{1}=1, \phi_{0}=1, \phi_{1}=1 \text {, at } y=0 \\
U_{0}=0, U_{1}=0, \theta_{0} \rightarrow 0, \theta_{1} \rightarrow 0, \phi_{0} \rightarrow 0, \phi_{1} \rightarrow 0 \text {, as } y \rightarrow \infty
\end{array}\right\}
$$

First we solve the equations (19) and (20) by using equation (21) then we get

$$
\begin{aligned}
& \phi_{0}=e^{-(\sqrt{S c K r}) y} \\
& \phi_{1}=e^{-(\sqrt{S c(K r+n)}) y}
\end{aligned}
$$

Now using multi parameter perturbation technique and assuming $E c<<1$.

$\left.\begin{array}{l}F_{0}=F_{00}+E c F_{01}+0(\varepsilon)^{2} \ldots \theta_{0}=\theta_{00}+E c \theta_{01}+0(\varepsilon)^{2} \ldots \\ F_{1}=F_{10}+E c F_{11}+0(\varepsilon)^{2} \ldots \theta_{1}=\theta_{10}+E c \theta_{11}+0(\varepsilon)^{2} \ldots . .\end{array}\right\}$

By using equations (24) in equations (15)-(18) and equating the coefficients of like powers of Ec neglecting those of $[E c]^{2}$ and $0[\varepsilon]^{2}$ we get the following set of differential equations,

$\left.\begin{array}{r}U_{0}=1, U_{1}=0, \theta_{0}=1 \theta_{1}=1, \phi_{0}=1, \phi_{1}=1, \text { at } y=0 \\ U_{0}=0, U_{1}=0, \theta_{0} \rightarrow 0, \theta_{1} \rightarrow 0, \phi_{0} \rightarrow 0, \phi_{1} \rightarrow 0 \text {, as } y \rightarrow \infty\end{array}\right\}$

$U_{00}^{\prime \prime}-\lambda U_{00}=-G_{r} \cos \psi \theta_{00}-G_{m} \cos \psi \phi_{0}$

$U_{01}^{\prime \prime}-\lambda U_{01}=-G_{r} \cos \psi \theta_{01}$

$U_{10}^{\prime \prime}-[\lambda+n i] U_{10}=-G_{r} \cos \psi \theta_{10}-G_{m} \cos \psi \phi_{1}$

$U_{11}^{\prime \prime}-[\lambda+n i] U_{11}=-G_{r} \cos \psi \theta_{11}$

$\theta_{10}^{\prime \prime}-\operatorname{Pr}[N+i n] \theta_{10}=-R_{a} \phi_{1} \operatorname{Pr}-\operatorname{Pr} \operatorname{Dr} \phi_{1}^{\prime \prime}$

$\theta_{11}^{\prime \prime}-\operatorname{Pr}[N+i n] \theta_{11}=-2 \operatorname{Pr} F_{00}^{\prime} F_{10}^{\prime}$

$\theta_{00}^{\prime \prime}-\operatorname{Pr} N \theta_{00}=-\operatorname{Pr} R_{a} \phi_{0}-\operatorname{Pr} \operatorname{Dr} \phi_{0}$

$\theta_{01}^{\prime \prime}-\operatorname{Pr} N \theta_{01}=-\operatorname{Pr}\left[U_{00}^{\prime}\right]\left[U_{00}^{\prime}\right]$

at $y=0 ; \Rightarrow\left\{\begin{array}{l}U_{00}=1, U_{01}=0, U_{10}=0, U_{11}=0, \\ \theta_{00}=1, \theta_{01}=0, \theta_{10}=1, \theta_{11}=0\end{array}\right.$

As $y \rightarrow \infty \Rightarrow\left\{\begin{array}{l}U_{00}=0, U_{01}=0, U_{10}=0, U_{11}=0, \\ \theta_{00}=0, \theta_{01}=0, \theta_{10}=0, \theta_{11}=0\end{array}\right\}$

\subsection{Velocity (F), Temperature $(\theta)$ and Concentration} $(\phi)$ : 


$$
\begin{aligned}
& F=\left[\left(F_{00}+E c F_{01}\right)+\varepsilon e^{\text {int }}\left(F_{10}+E c F_{11}\right)\right] \\
& \theta=\left[\left(\theta_{00}+E c \theta_{01}\right)+\varepsilon e^{\text {int }}\left(\theta_{10}+E c \theta_{11}\right)\right] \\
& \phi=\phi_{0}+\varepsilon e^{\text {int }} \phi_{1}
\end{aligned}
$$

\section{Results and Discussion:}

In the present study we have to select $\mathrm{t}=1.0, \mathrm{n}=0.5, \epsilon=0.03$, $\eta=0.03 \xi=0.03$ while $\mathrm{Gm}, \gamma, \mathrm{M}, \mathrm{Ra}, \mathrm{Ec}, \beta_{i}, \beta_{e}$ and Dr are varied over a range, which listed in the figure. Figure 1:, Figure 2: and Figure 3: from this figures reflects that for different values of inclination of magnetic field $\psi$, Dufour effect Dr and ion-slip parameter $\beta_{i}$ increases then it lead to velocity profile increases. Figure 4: Figure 5: Depict the effect of Dufour affect Dr and Eckert number Ec on the temperature profile. It is observed that temperature scores increases with the increase of Dr and Ec. Figure 9: the velocity profile decreases with an increase in $\beta_{e}$.

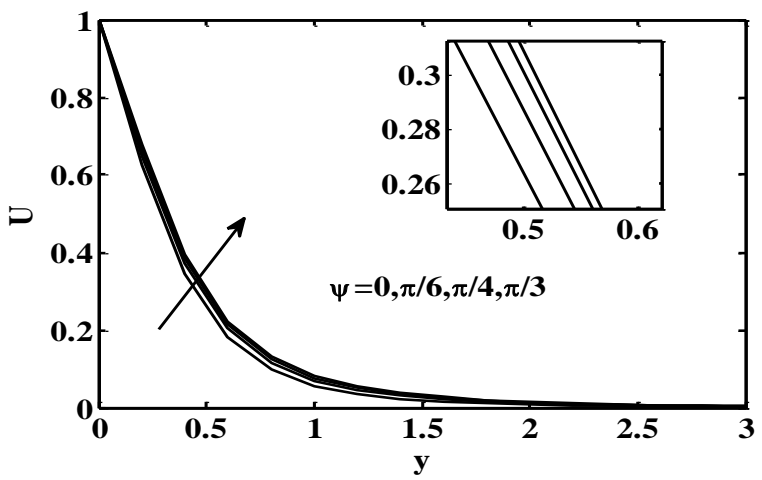

Fig 1: Effect of $\psi$ for different values on Velocity

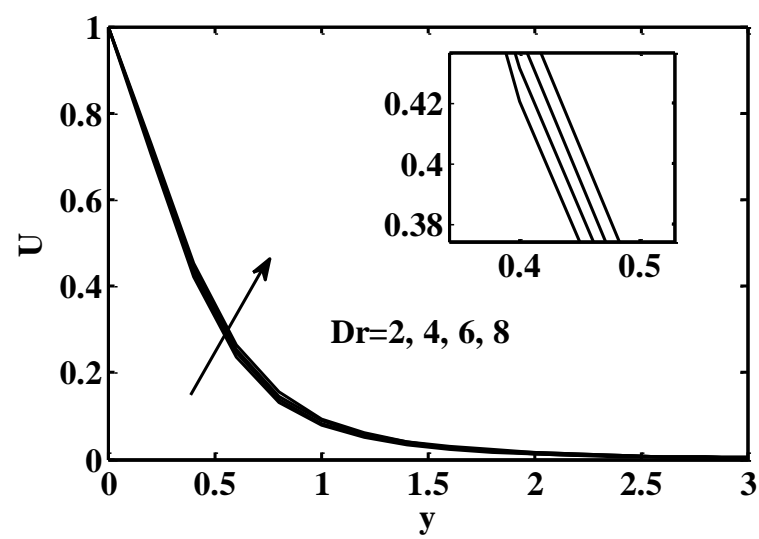

Fig 2: Effect of Dr for different values on Velocity

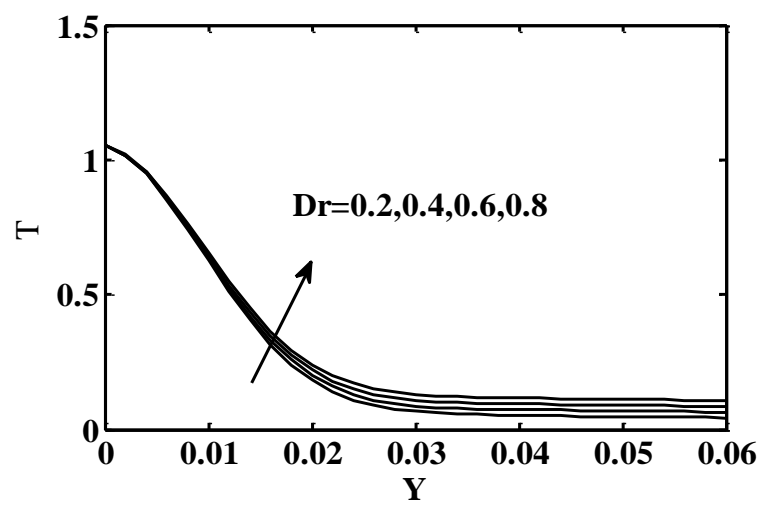

Fig 3: Effect of Dr for different values on Temperature

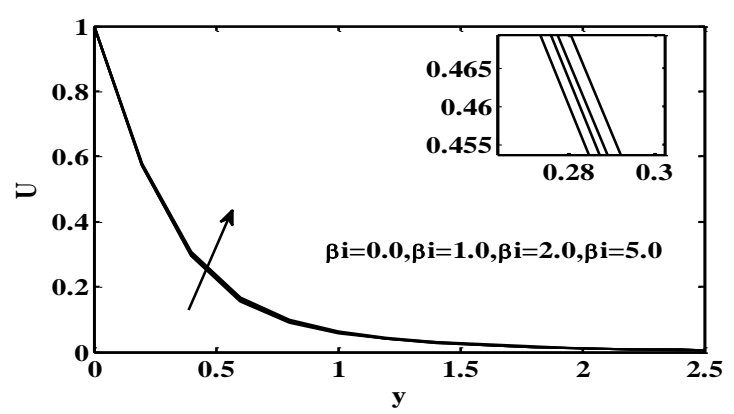

Fig 4: Effect of $\beta_{i}$ for different values on Velocity

From the figure 7: for different values of Pr increase then it leads to decrease in temperature. From the Figure 8: For different values $\mathrm{R}$ increases then it lead to increases in temperature

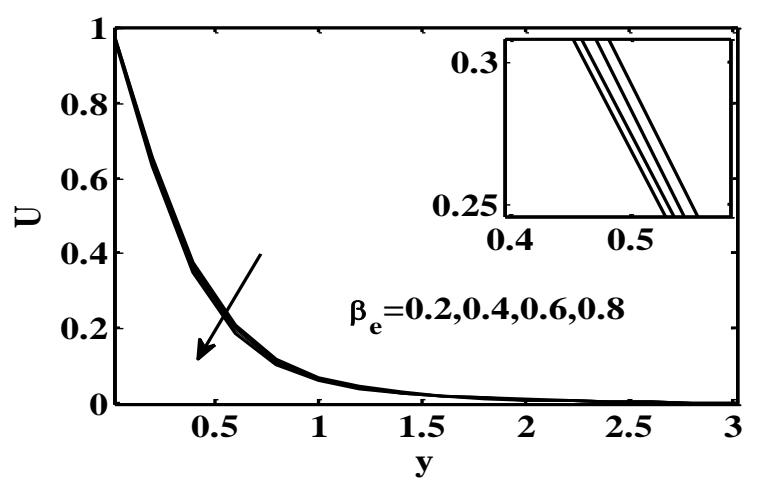

Fig 5: Effect of $\beta_{e}$ for different values on Velocity

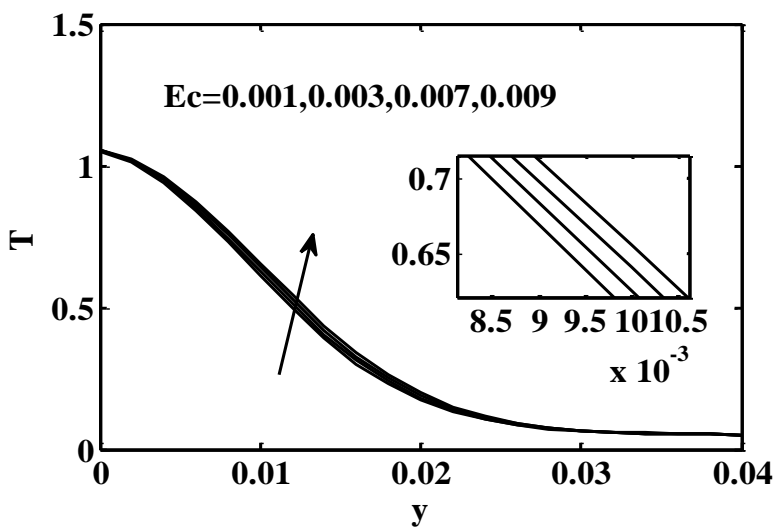

Fig 6: Effect of Ec for different values on Temperature

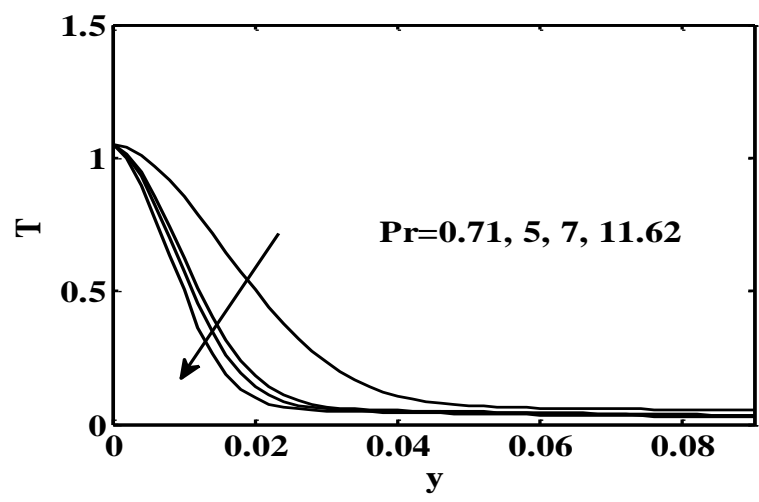

Fig 7: Effect of Pr for different values on Temperature 


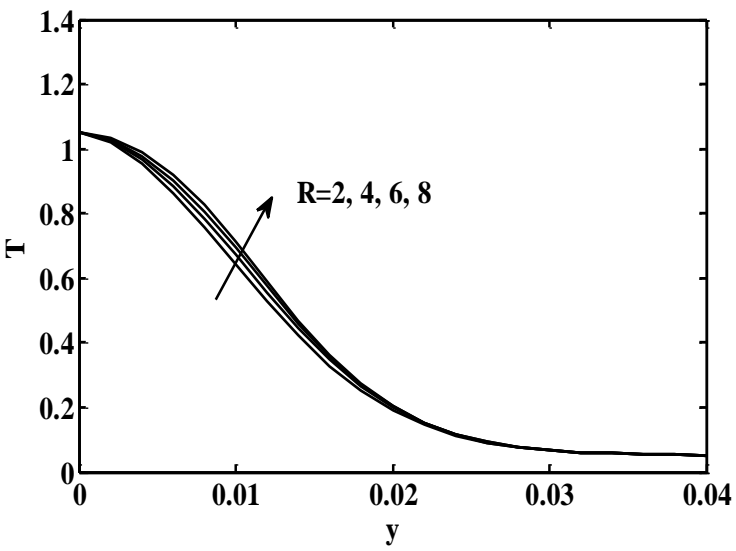

Fig 8: Effect of $\mathrm{R}$ for different values on Temperature

\section{Conclusions:}

The dimensionless governing equations for this investigation are solved analytically by using multiple regular perturbation law. The effects of different parameters on velocity, temperature and concentration fields are discussed in detail. As temperature profile increases with the increase of Eckert number Ec. Velocity increases with increase in inclined angle $\psi$.As the Ion-slip parameter $\beta_{i}$ increases the Velocity profiles increases and velocity decrease with increases of Hall current parameter $\beta_{e}$.As the Dufour effect parameter Dr Increases the velocity and temperature increases.

\section{References:}

[1] Shankar Goud, (2017) Finite element solution on effects of viscous dissipation and diffusion thermo on unsteady Magneto hydrodynamic flow past an impulsively started inclined oscillating plate with mass diffusion and variable temperature. Int. research J. eng and tech Vol.4,no.1.

[2] Alivene, Sreevani. (2017) Effect of Hall current, thermal radiation, dissipation and chemical reaction on hydro magnetic non-Darcy mixed convective heat and mass transfer flow past a stretching sheet in the presence of heat sources. Adv in phy theo and appl. Vol. 61.

[3] Subhakanthi, (2016) Chemical reaction effects on Heat and Mass Transfer in MHD boundary layer Flow past an Inclined Plate with viscous dissipation and radiation in Porous Medium. Int J. of eng and management Research. Vol. 6, no. 3, pp. 447-459.

[4] Viswanatha Reddy, (2017) Chemical reaction and thermal radiation effect on unsteady MHD free convection flow past an inclined moving plate with TGHS Imperial J. of Interdisciplinary Research. Vol.3, no. 1, pp. 2454-1362.

[5] Rapt and Gaurav Kumar. (2017) Effects of hall current and chemical reaction on MHD flow through porous medium past an oscillating inclined plate with variable temperature and mass diffusion. European Journal of Adv in Eng and Technology, Vol.4, no. 1, pp. 5663.

[6] Rajput and Gaurav. (2017) Chemical reaction effect on unsteady MHD flow past an impulsively started inclined plate with variable temperature and mass diffusion in the presence of Hall Current. Jordan J. of Mech. and Industrial Eng. Vol. 11, pp. 41-49.

[7] Rajput and Gaurav, (2016) Unsteady MHD Flow in Porous Media Past Over exponentially accelerated inclined plate with variable wall temperature and mass transfer along with Hall current, Int., J., of Eng, Sci and Technology. Vol. 8, no.2, pp. 1-10.

[8] Jithender Reddy, Srinivasa Raju. Anand Rao,(2016) Thermal diffusion and diffusion thermo effects on unsteady MHD fluid flow past a moving vertical plate embedded in porous medium in the presence of hall current and rotating system, Transactions of a. Razmadze mathematical institute journal, Vol.170, pp. $243-265$

[9] Mohammad Shah Alam, Ashutose Saha.(2015) Numerical study of one dimensional free convection heat and mass transfer of fresh and salt water flow on an infinite inclined plate with hall current and constant heat flux in presence of magnetic field. International Journal of Thermal Energy and Applications. Vol. 1, no. 2, pp. 12-25.

[10] Sivaiah K. Jayarami Reddy. (2016) Unsteady MHD heat and mass transfer flow of a radiating fluid past an accelerated inclined porous plate with hall current. IOSR Journal of Mathematics. Vol. 12, no. 5, pp. 24-36.

Nomenclature:

$\mathrm{u} \quad$ Velocity component

$\gamma \quad$ Permeability of porous medium

$T_{w} \quad$ Temperature at the plate

$T_{\infty} \quad$ Temperature outside of the boundary lyre(K)

$C_{w} \quad$ Concentration at the plate

$C_{\infty} \quad$ Concentration outside of the plate $\left(\mathrm{kg} \cdot \mathrm{m}^{-3}\right)$

$\Omega \quad$ Rotational velocity component

U Dimensionless primary velocity

W Dimensionless secondary velocity

$T^{*}$

Dimensionless fluid temperature

$C^{*} \quad$ Dimensionless fluid concentration

$\psi \quad$ Inclined angle

B Magnetic field

$B_{0} \quad$ Magnetic component(A.m-1)

$U_{0} \quad$ Uniform velocity

Gr Grashof number

Gr Modified Grashof number

$n^{*} \quad$ Mining

Ec Eckret number

R Rotational parameter

$\eta \quad$ Radiation parameter

$\tau_{w} \quad$ Skin-friction coefficient

$\xi$

Free stream condition

electron charge $(\mathrm{C})$

$\beta_{e} \quad$ Hall parameter

$\beta_{i} \quad$ Ion-slip parameter

Magnetic permeability of the porous medium

Thermal expansion co-efficient(K-1)

Concentration expansion co-efficient(m3.kg-1)

Heat source parameter

Kinematic velocity( m2.S-1)

Density of the fluid (kg.m-3)

$C_{p}$

$D_{m}$

$Q_{0}$

$K_{r}$

Electrical conductivity $\left(\Omega^{-1} m^{-1}\right)$

$$
\text { Thermal conductivity(W.m-1 .K-1) }
$$

$p$

Specific heat at constant pressure (J. kg -1.K)

Co-efficient of mass diffusivity(m2.S-1)

$$
\text { Heat absorption quantity }
$$

Chemical reaction parameter(m.S-1)

$$
\text { Mean fluid temperature(K) }
$$

\section{Prandtl number}

Dufour number

Schmidt number

Arbitrary constant 
$\mathrm{Ra}$ Thermal radiation parameter

$\alpha_{e} \quad=1+\beta_{e} \beta_{i}$

$N_{u} \quad$ Nusselt number

$t^{*} \quad$ Dimensional time(S)

$q_{r}^{*} \quad$ Radiation heat flux density(W.m-2)

g acceleration due to gravity(m.S-2)

$\mathrm{x}, \mathrm{y} \quad$ Cartesian co-ordinates 